\title{
Trauma and post-traumatic stress disorder in a drug treatment community service
}

\author{
Martina Reynolds, ${ }^{1}$ Kate Hinchliffe, ${ }^{1}$ Victor Asamoah, ${ }^{2}$ Christos Kouimtsidis ${ }^{2}$
}

The Psychiatrist (2011), 35, 256-260, doi: 10.1192/pb.bp.110.030379

${ }^{1}$ Brunel University, Uxbridge;

${ }^{2}$ Hertfordshire Partnership NHS

Foundation Trust, Hemel Hempstead

Correspondence to Christos

Kouimtsidis

(drckouimtsidis@hotmail.com)

First received 11 Mar 2010, final revision 29 Nov 2010, accepted 13 Jan 2011

\begin{abstract}
Aims and method A cross-sectional study aiming to assess the prevalence of trauma and post-traumatic stress disorder (PTSD) in a community substitution treatment sample, and to assess and compare the characteristics of traumatic experience, substance use, and psychological and social factors in those with and without PTSD. All assessments were completed during the interview which took approximately $1.5 \mathrm{~h}$.
\end{abstract}

Results The prevalence for current PTSD was $26.2 \%$ and for lifetime PTSD $42.9 \%$. Traumatic experiences were extremely common, with two or more reported by $92.9 \%$ of the sample. The two groups differed significantly on the majority of psychological functioning and social variables, with women experiencing higher rates of PTSD and the non-PTSD group having lower rates of psychological impairment.

Clinical implications The research supported findings from previous studies. The very high incidence of traumatic experiences has not been reported before. Training and information about trauma and PTSD for substance misuse workers are therefore necessary so that PTSD can be more easily detected and treated.

Declaration of interest None.
A large body of research, mostly from the USA, documents the relationship between trauma, post-traumatic stress disorder (PTSD) and substance use disorder in a range of different populations. ${ }^{1-3}$ Relatively high rates of comorbid PTSD have been reported and associated with poor treatment outcome and prognosis. ${ }^{4-7}$ In the USA, rates of comorbid PTSD and substance misuse range from 19 to $35 \%$, and comorbid alcohol misuse from 36 to $52 \% .^{1,2}$ In individuals with substance use disorder, lifetime PTSD has been reported in $30-58 \%$, and current PTSD in $20-38 \%{ }^{8,9}$

Research examining associations between PTSD symptoms and substance use disorder suggests that substance misuse may be motivated by a desire to control the intrusion and arousal symptom groups, or that these symptoms may be exacerbated by substance misuse. ${ }^{10-12}$ The picture is confounded by the actual phenomenology of substance misuse, where different classes of drugs affect individuals differently and are taken at different stages throughout an individual's drug use history, therefore complicating the presenting comorbidity. ${ }^{10,13}$ Such findings have important implications for the comorbid diagnosis and treatment of substance use disorder and PTSD. Substantial evidence supports the view that people with a comorbid diagnosis of PTSD and substance use disorder have more severe symptoms and other related problems than those without this comorbidity. ${ }^{14,15}$

There is relatively little in the way of published studies exploring this phenomenon in the UK. Reynolds et $a l^{16}$ found a strong connection between substance use disorder, trauma and PTSD. They reported prevalence rates of comorbid PTSD and substance use disorder of $38.5 \%$ for current PTSD and 51.9\% for lifetime PTSD, and the reported rate of traumatic experiences was $94.2 \%$. Other findings of note were higher rates of sexual assault, crime-related events, higher levels of current distress associated with the target trauma (most distressing trauma as identified by participants using the Trauma History Questionnaire ${ }^{17}$ ), and a larger impact of the trauma on work, family and social relationships in the PTSD group. As this was an in-patient population, it would be expected that they would be more severely affected by other problems and comorbid psychopathology; therefore it is important to replicate the study with a community population receiving substitution treatment.

The aim of our study was twofold: (1) to assess the prevalence of trauma and PTSD in a community sample; and (2) to assess and compare characteristics of traumatic experience, substance use, and psychological and social factors in those with and without PTSD.

\section{Method}

The interview schedule and assessments used by Reynolds et $a l^{16}$ were used to enable a comparison of the findings. Individuals attending the community drug and alcohol team were approached by the researcher before or after their regular weekly, 2-weekly or monthly appointment and an 
interview was arranged. All assessments were completed during the interview which took approximately $1.5 \mathrm{~h}$. Participants were reimbursed for expenses only. Written consent was obtained following a full explanation of the study in accordance with National Research Ethics Service guidelines. All participants were made aware of their right to decline or withdraw consent at any time without explanation and were informed that their decision on whether or not to participate in the study would not affect their treatment.

\section{Participants}

Participants for the community sample were recruited from service users attending a community drug and alcohol team who serve a large urban and rural community of approximately 250000 people just outside London. All individuals were on substitution treatment with either methadone or buprenorphine, and were approached during a 5-week period. The researchers had no knowledge of the users' histories prior to interviewing, so all those who participated were representative of the team population in general. All users were over 18 years of age, and only those with current severe mental illnesses (e.g. schizophrenia) and/or brain damage or other organic impairment which would impair their ability to participate were excluded.

\section{Assessment and questionnaires}

Demographic details, trauma exposure, PTSD, substance use and associated problems, and other comorbid disorders were assessed using the semi-structured interview schedule and questionnaires as described below. A full description of these assessments can be found in Reynolds et al. ${ }^{16}$

1. Addiction Severity Index (ASI). ${ }^{18}$ An interview to detect and measure severity of problems in seven areas (ethnic/ cultural, medical, employment/support, drug/alcohol use, legal status, family/social relationships and psychiatric). Severity is indicated by a composite score for each area derived from responses to specific questions in each section.

2. Trauma History Questionnaire (THQ). ${ }^{17}$ This consists of 24 items addressing experience, number of times and age at occurrence with respect to a range of traumatic events in three areas: crime-related events, general disaster and trauma, and unwanted physical and sexual experiences. Additional ratings included distress at the time of the trauma and distress currently. After completing the THQ, participants were asked to identify the trauma that they felt was the most distressing and had the worst impact on them overall ('target trauma').

3. PTSD Symptom Scale - Interview Version (PSS-I). ${ }^{19}$ This is a 17-item scale designed to assess PTSD symptomatology; it consists of questions pertaining to the symptoms in the re-experiencing, avoidance and arousal categories of PTSD. This interview was completed in relation to the trauma identified as the most distressing overall in the THQ (target trauma). A diagnosis of PTSD is made on the basis of meeting the DSM-IV-TR ${ }^{20}$ criteria for PTSD. The items endorsed on the scale can also be summed to give a total score for symptom groups B, C and D on DSM-IV-TR. A score of 17 or more indicated a cut-off for PTSD.
4. Brief Symptom Inventory (BSI). ${ }^{21}$ This is a 53 -item selfreport symptom inventory which reflects the 9 primary symptom dimensions (somatisation, obsessive-compulsive, interpersonal sensitivity, depression, anxiety, hostility, phobic anxiety, paranoid ideation and psychoticism) of the 90-item Symptom Checklist - Revised (SCL-90-R) ${ }^{22}$ and is designed to assess the psychological symptom status of psychiatric and medical patients.

5. Traumatic memory and trauma characteristics interview. ${ }^{16}$ This part of the study will be reported separately.

6. Social functioning was assessed by asking participants to rate on a visual analogue scale, where $0=$ not at all and $10=$ very much so, how much the trauma-associated problems had interfered with their work, social life/ leisure activities, and family life/home responsibilities.

\section{Case-note review}

A case-note review was undertaken regarding:

- whether reference was made to any past traumas in the case notes (for the purpose of this study, reference to items listed in the THQ was used as a guide for definition of a trauma; answers were coded yes/no)

- nature of traumas documented

- number of traumas documented

- whether a PTSD diagnosis was made/referred to (answers were coded yes/no)

- if a PTSD diagnosis was made, whether the patient was referred for treatment (answers were coded yes/no).

The notes were screened for all traumas documented.

\section{Results}

\section{Analysis}

All analyses were undertaken using SPSS for Windows, version 10. Associations between categorical variables were investigated using chi-squared tests, and when invalid, Fisher's test was employed. For scaled variables, one-way analysis of variance was used and equivalent nonparametric procedures were used for data that violated assumptions of normal distribution.

\section{Demographic data}

Out of 92 individuals in treatment, 86 attended the service during the study period. From those, 70 individuals were approached: 28 refused to participate and 42 were interviewed, giving a conversion rate of $46 \%$. Twentyseven individuals $(64.3 \%)$ were male. The mean age of the males was 36.3 years (s.d. $=10.7)$ and the mean age of the females was 36.5 years (s.d.=6.7); the difference was not significant $(t=-0.066$, d.f. $=40, P>0.10)$. Forty individuals (95.2\%) in the sample were White. Twenty-seven (64.3\%) had a qualification, skill or profession. In the 3 years prior to the interview, 28 (66.7\%) were unemployed, 11 (26.2\%) were working full- or part-time, and $3(7.1 \%)$ were retired, ill or disabled. With regard to living arrangements, 15 (35.7\%) lived alone, 10 (23.8\%) with a partner and children, 8 (19\%) with a partner alone, $3(7.1 \%)$ alone with their children, 2 (4.8\%) with their parents, $2(4.8 \%)$ with a family member and $1(4.2 \%)$ with friends. 


\section{PTSD}

Eleven participants $(26.2 \%)$ met DSM-IV-TR ${ }^{20}$ criteria for PTSD at the time of the interview, 4 (9.5\%) were categorised as having a partial PTSD because they did not meet the full criteria for a diagnosis, and 27 (64.3\%) did not meet criteria for the diagnosis. Eighteen participants (42.9\%) met DSMIV-TR criteria for a lifetime PTSD diagnosis, 4 (9.5\%) for a lifetime diagnosis for partial PTSD and 20 (47.6\%) did not meet criteria for the diagnosis. The subsequent analyses in this report will involve comparisons between the 'PTSD group' and the 'non-PTSD group'. The latter includes those who did not meet criteria for PTSD diagnosis and also those who met criteria for a partial diagnosis described here, as in Reynolds et al. ${ }^{16}$ The 'partial PTSD' group were included in the non-PTSD group for the main analysis because on the whole their scores were within the range of the latter.

\section{Trauma}

Thirty-nine individuals (92.9\%) reported two or more DSMIV-TR criterion A events for PTSD and the remaining three (7.1\%) reported one event. Target traumas for the PTSD and non-PTSD group were identified for the purpose of assessing post-traumatic symptomatology. Thirty-five (85.4\%) of target traumas reported met PTSD criterion A definition. The PTSD group experienced significantly more criterion A traumatic events than the non-PTSD group (10.1 and 6.3 respectively; $t=-2.67 ; P<0.05$ ), with all traumas reported by the PTSD group meeting criterion A specification. The target trauma (as identified by participants in a short interview following completion of the THQ) occurred more than 12 months before the interview for 38 participants (92.7\%): 11 (100\%) of the PTSD group and 27 (87\%) of the non-PTSD group. Six individuals (54\%) in the PTSD group and ten (32\%) in the non-PTSD group were under the age of 17 years when the target trauma occurred. Seven of the eight sexual assaults reported as target traumas occurred when the participants were under 17 years.

The THQ traumas were subdivided into categories and the following rates were reported: experiencing mugging, robbery and break-in, PTSD group 11 (100\%), non-PTSD group $26(86.7 \%)$; serious accidents, natural or manmade disasters, PTSD group 9 (81.8\%), non-PTSD group 21 (70\%); witnessing serious injury or death, PTSD group 7 (63.6\%), non-PTSD group 19 (63.3\%); traumatic bereavement, PTSD group 9 (81.8\%), non-PTSD group 23 (76.7\%); miscellaneous trauma, PTSD group 5 (45.5\%), non-PTSD group 10 (33.3\%); sexual assault, PTSD group 8 (72.7\%), non-PTSD group 7 (23.3\%); and threatened or actual physical assault, PTSD group 9 (81.1\%), non-PTSD group 18 (60\%).

\section{Trauma characteristics and PTSD diagnosis}

Significantly more of the PTSD group than the non-PTSD group reported sexual assaults (PTSD 8/11, non-PTSD 7/30; $\left.\chi^{2}(1)=8.46, P<0.005\right)$. Of these, $2 / 11$ of the PTSD group and $4 / 30$ of the non-PTSD group reported multiple sexual assaults. With lifetime PTSD as dependent variable, significantly more individuals in the PTSD group than in the non-PTSD group reported experiences of mugging, robbery and break-in (PTSD 19/19, non-PTSD 18/22; $\chi^{2}(1)=3.83$, $P<0.05$ ), and sexual assault (PTSD 12/19, non-PTSD 3/22; $\left.\chi^{2}(1)=10.78, P<0.001\right)$. The groups did not differ in the time since the target trauma occurred (Fisher's exact $P>0.10$ ).

The PTSD group reported a higher level of current distress associated with the target trauma than the nonPTSD group (mean PTSD 7.7 (mean rank 32.6), mean nonPTSD 3.4 (mean rank 16.7); $Z=-3.78, P<0.0005$ ), and a higher level of distress at the time of the trauma (mean PTSD 9.8 (mean rank 30.1), mean non-PTSD 7.9 (mean rank 17.6); $Z=-3.15, P<0.005)$. Current distress levels were significantly lower than those reported at the time of the trauma for both groups, but particularly so for the nonPTSD group: PTSD mean rank $3.5, Z=-2.23, P<0.05$; nonPTSD mean rank $13, Z=4.38, P<0.0005$.

There was a significant difference in gender for current PTSD (females 7/15, males 4/27; $\chi^{2}(1)=4.74$, $P<0.05$ ) and lifetime PTSD (females 10/15, males 9/27; $\left.\chi^{2}(1)=3.93, P>0.05\right)$, with a higher rate of PTSD in females. The PTSD group rated the social impact of the trauma on their lives significantly higher than the nonPTSD group in the following areas: impact on work (mean PTSD 5.64 (mean rank 29.9), mean non-PTSD 0.83 (mean rank 17.7); $Z=-3.24, P<0.001)$, impact on social life (mean PTSD 7.09 (mean rank 32.9), mean non-PTSD 1.83 (mean rank 16.6); $Z=-3.99, P<0.0005$ ), and impact on family responsibilities (mean PTSD 6.73 (mean rank 32.1), mean non-PTSD 2.00 (mean rank 16.9); $Z=-3.71$, $P<0.0005)$.

\section{Addiction severity}

Twenty-two participants $(52.4 \%)$ stated that heroin was a substance they misused most, nine (21.4\%) reported polydrug use, four (9.5\%) cocaine (as part of polydrug use), two (4.8\%) other opiates/analgesics, two (4.8\%) methadone, two (4.8\%) alcohol (as part of polydrug use), and one (2.4\%) did not specify a particular substance.

The PTSD group had significantly higher ratings than the non-PTSD group for the ASI medical, family and psychiatric composite scores. There were no other significant differences in ASI scores (Table 1).

Further analysis of addiction variables showed that the PTSD group reported significantly more lifetime years of hallucinogen use (mean PTSD 4.64 (mean rank 27.6), mean non-PTSD 1.27 (mean rank 18.6); $Z=-2.27, P<0.05$ ), and the non-PTSD group had significantly less lifetime years of alcohol use (mean PTSD 6.91 (mean rank 13.7), mean nonPTSD 15.37 (mean rank 23.7); $Z=-2.37, P<0.05$ ). No other significant differences were found between current PTSD and non-PTSD groups on addiction variables.

The same comparisons were made for lifetime PTSD. Here the PTSD group reported significantly more drug overdoses (mean PTSD 17.4 (mean rank 25.18), mean nonPTSD 9.60 (mean rank 17.39); $Z=-2.26, P<0.05$ ) and more lifetime amphetamine use (mean PTSD 5.16 (mean rank 25.3), mean non-PTSD 2.86 (mean rank 17.3); $Z=-2.16$, $P<0.05)$ than the non-PTSD group.

\section{Psychiatric symptoms}

There were significant differences between the PTSD and non-PTSD groups for all BSI symptom groups and totals, in which the scores of the PTSD group were significantly higher than the non-PTSD group $(P<0.05$ to $P<0.0005)$. 


\begin{tabular}{l} 
Table 1 Addiction Severity Index (ASI): means and group differences for current post-traumatic stress disorder (PTSD) \\
diagnosis \\
\cline { 2 - 3 } Variable ASI composite scores
\end{tabular}

ns, not significant.

${ }^{\star \star} P<0.005 ;{ }^{* \star \star} P<0.0005$.

\begin{tabular}{|c|c|c|c|c|}
\hline \multirow[b]{2}{*}{ Variable BSI symptom groups } & \multicolumn{2}{|c|}{ Mean (s.d.) } & \multirow[b]{2}{*}{ Test statistic } & \multirow[b]{2}{*}{$P$} \\
\hline & $\begin{array}{l}\text { PTSD } \\
N=11\end{array}$ & $\begin{array}{c}\text { Non-PTSD } \\
N=27\end{array}$ & & \\
\hline Somatisation & $1.84(1.13)$ & $1.00(0.67)$ & $F(1,37)=8.07$ & ** \\
\hline Obsessive-compulsive & $2.47(1.02)$ & $0.89(0.76)$ & $F(1,37)=27.38$ & $\star * * *$ \\
\hline Interpersonal sensitivity & $2.25(1.09)$ & $0.74(0.71)$ & $F(1,37)=25.57$ & $* * * *$ \\
\hline Depression & $2.48(1.22)$ & $0.91(0.88)$ & $F(1,37)=19.79$ & 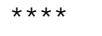 \\
\hline Anxiety & $1.92(1.11)$ & $0.78(0.74)$ & $F(1,37)=13.84$ & $* * *$ \\
\hline Hostility & $1.53(1.21)$ & $0.67(0.77)$ & $F(1,37)=6.88$ & * \\
\hline Phobic anxiety & $1.87(1.35)$ & $0.44(0.68)$ & $F(1,37)=18.90$ & $\star * * *$ \\
\hline Paranoid ideation & $2.25(1.22)$ & $0.91(0.68)$ & $F(1,37)=18.81$ & $\star * * *$ \\
\hline Psychoticism & $1.82(1.26)$ & $0.57(0.65)$ & $F(1,37)=16.16$ & $\star * * *$ \\
\hline Global Severity Index & $1.92(0.96)$ & $0.75(0.58)$ & $F(1,37)=10.67$ & $* * * *$ \\
\hline BSI positive symptom total & $40.64(13.37)$ & $24.11(14.05)$ & $F(1,37)=11.14$ & ** \\
\hline BSI positive symptom distress index & $2.52(0.77)$ & $1.66(0.57)$ & $F(1,37)=14.55$ & $* * *$ \\
\hline
\end{tabular}

${ }^{\star} P<0.05 ;{ }^{* \star} P<0.01 ;{ }^{* \star *} P<0.001 ;{ }^{\star \star \star \star} P<0.0005$.

The BSI symptom group and totals for the current PTSD group are presented in Table 2.

\section{Case-note review}

The case-note review showed that traumas were referred to in $34(85 \%)$ of the case notes of those who reported traumas during the interview. Traumas documented included: family/ personal issues $(n=5)$, traumatic bereavement $(n=13)$, assault $(n=5)$, personal injury $(n=3)$ and sexual abuse $(n=5)$. No PTSD diagnoses or referrals were documented.

\section{Discussion}

In this out-patient study the prevalence rates were $26.2 \%$ for current PTSD and $42.9 \%$ for lifetime PTSD, lower than the $38.5 \%$ and $51.9 \%$ respectively found by Reynolds et $a l^{16}$ in an in-patient sample. The rates are broadly similar to those reported in US studies. ${ }^{1,2,8,9}$ Despite this, rates of reported trauma were high, with $92.9 \%$ of participants reporting two or more DSM-IV-TR criterion A events for PTSD, and all reporting at least one event. The PTSD group experienced significantly more criterion A events than the non-PTSD group.

Further exploration of traumas experienced showed that THQ traumas reported across the different categories ranged from 45.5 to $100 \%$ in the PTSD group and from 23.3 to $86.7 \%$ in the non-PTSD group, with no significant differences apart from sexual assault, rates of which were considerably higher in the PTSD group (72.2\%) than the non-PTSD group (23.3\%). There was also a tendency for reported rates of traumas in the other categories to be higher in the PTSD group. For $92.7 \%$ of the total sample and $100 \%$ of the PTSD group, the target trauma occurred more than 12 months before the interview. Seven of the eight sexual assaults reported as target traumas occurred when participants were under 17 years of age. Rates of current and lifetime PTSD were significantly higher in females.

It is important to note that the assessment tools used in the study, although validated in the general population and other psychiatric populations, and used in previous similar studies in substance misuse, are not validated in substance misuse populations per se. Another limitation of 
this study is that multivariate analysis was not used so the findings may not be independent of each other.

These findings suggest that although traumatic experiences are extremely common in substance use disorder populations, only a proportion (about 25\%) will have a PTSD diagnosis. It may be that some individuals are more vulnerable, and even though they have not experienced more traumas, or their traumas were not more severe, the impact of such events psychologically and socially was considerably more severe, as indicated by the significantly higher levels of retrospective and current distress associated with the target trauma, and the higher social impact in terms of work, social life and family responsibility, reported by the PTSD group. It could be argued that these results could be expected considering the lifestyle of the population with opioid dependence, who represent the more severe end of substance misuse. It is important to note that the design of this study did not assess the causal relationship between trauma experience, PTSD and substance misuse, therefore we cannot infer whether or not substance misuse was developed as a way to cope with the effects of trauma experienced by individuals. A qualitative study could investigate better the relationship between trauma and substance misuse and could add to the interpretation of the results reported here.

What becomes apparent upon revisiting the findings of the Reynolds et al study ${ }^{16}$ is that relatively few and small differences in addiction, general psychological and social variables between the PTSD and non-PTSD groups were found. To the contrary, the PTSD and non-PTSD groups in our study differed significantly on the majority of the general psychological functioning and social variables, including the ASI medical, family and psychiatric composite scores, all BSI symptom groups and totals, impact on social, work and family life, as well as incidence of accidental overdoses and comorbid misuse of alcohol, suggesting that the PTSD group in this study in many ways experience significantly worse psychological and social impairment than the non-PTSD group. These findings resemble those of previous studies. ${ }^{10,14,15}$

This study did not examine directly the effect of the psychological and social impairment on the response to treatment for substance use disorder, but the findings support previous findings suggesting that assessment of individuals attending substance use clinics for trauma and PTSD may be very useful for a number of reasons. Although traumas were referred to in a large proportion of the case notes, no formal assessments were undertaken and no referrals were made. Providing training and information about trauma and PTSD for substance misuse workers is therefore necessary so that PTSD can be more easily detected and treated, and underlying problems can be addressed, which might optimise treatment response and limit healthcare costs in the long term.

\section{About the authors}

Martina Reynolds, senior lecturer, Kate Hinchliffe, MSc student, School of Social Sciences, Brunel University, Uxbridge, Middlesex, UK; Victor Asamoah, specialist registrar, Christos Kouimtsidis, consultant psychiatrist, Community Drug and Alcohol Team, Hertfordshire Partnership NHS Foundation Trust, Hemel Hempstead, UK.

\section{References}

1 Kessler RC, Sonnega A, Bromet E, Hughes M, Nelson CB. Post traumatic stress disorder in the National Co-morbidity Survey. Arch Gen Psychiatry 1995; 52: 1048-60.

2 Kilpatrick DG, Acierno R, Saunders B, Resnick H, Best C, Schnurr P. Risk factors for adolescent substance abuse and dependence: data from a national sample. J Consult Clin Psychol 2000; 68: 19-30.

3 Creamer M, Burgess P, McFarlane A. Post-traumatic stress disorder: findings from the Australian National Survey of Mental Health and Well-being. Psychol Med 2001; 31: 1237-47.

4 Brown PJ, Recupero PR, Stout R. PTSD substance abuse co-morbidity and treatment utilization. Addict Behav 1995; 20: 251-4.

5 Najavits LM, Gastfriend DR, Barber JP, Reif S, Muenz LR, Blaine J, et al. Cocaine dependence with and without PTSD among subjects in the National Institute on Drug Abuse Collaborative Cocaine Treatment Study. Am J Psychiatry 1998; 155: 214-9.

6 Ouimette PC, Ahrens C, Moos RH, Finney JW. Post-traumatic stress disorder in substance abuse patients: relationship to 1-year posttreatment outcomes. Psychol Addict Behav 1997; 11: 34-47.

7 Schäfer I, Najavits LM. Clinical challenges in the treatment of patients with posttraumatic stress disorder and substance abuse. Curr Opin Psychiatry 2007; 20: 614-8.

8 Cottler LB, Compton III WM, Mager D, Spitznagel EL, Janca A Posttraumatic stress disorder among substance users from the general population. Am J Psychiatry 1992; 149: 664-70.

9 Deykin EY, Buka SL. Prevalence and risk factors for post-traumatic stress disorder among chemically dependent adolescents. Am Psychiatry 1997; 154: 752-7.

10 Chilcoat HD, Breslau N. Post-traumatic stress disorder and drug disorders: testing causal pathways. Arch Gen Psychiatry 1998; 55: 913-7.

11 Triffelman EG, Marmar CR, Delucchi KL, Ronfeldt H. Childhood trauma and post-traumatic stress disorder in substance abuse inpatients. J Nerv Ment Dis 1995; 183: 172-6.

12 McFall ME, Mackay PW, Donovan DM. Combat-related post traumatic stress disorder and severity of substance abuse in Vietnam veterans. J Stud Alcohol 1992; 53: 357-63.

13 Stewart SH, Pihl RO, Conrod P, Dongier M. Functional association among trauma, PTSD and substance-related disorders. Addict Behav 1998; 23: 797-812.

14 Ouimette PC, Goodwin E, Brown PJ. Health and well being of substance use disorder patients with and without posttraumatic stress disorder. Addict Behav 2006; 31: 1415-23.

15 Najavits LM, Harned MS, Gallop RJ, Butler SF, Barber JP, Thase ME, et al Six-month treatment outcomes of cocaine-dependent patients with and without PTSD in a multisite national trial. J Stud Alcohol Drug 2007; 68: 353-61.

16 Reynolds M, Mezey G, Chapman M, Wheeler M, Drummond C, Baldacchino A. Co-morbid post-traumatic stress disorder in a substance misusing clinical population. Drug Alcohol Depend 2005; 77: 251-8.

17 Green BL. Psychometric review of Trauma History Questionnaire (Selfreport). In Measurement of Stress, Trauma and Adaptation (eds $\mathrm{BH}$ Stamm, EM Varra): 366-88. Sidran, 1993.

18 McLellan AT, Alterman Al, Cacciola J, Metzger D, O'Brien CP. The fifth edition of the Addiction Severity Index. J Subst Abuse Treat 1992; 180: 101-10.

19 Foa EB, Riggs DS, Dancu CV, Rothbaum BO. Reliability and validity of a brief instrument for assessing post-traumatic stress disorder. J Trauma Stress 1993; 6: 459-73.

20 American Psychiatric Association. Diagnostic and Statistical Manual of Mental Disorders, Fourth Edition, Text Revision (DSM-IV-TR). APA, 2000.

21 Derogatis LR, Melisaratos N. The Brief Symptom Inventory: an introductory report. Psychol Med 1975; 13: 595-605.

22 Derogatis LR. Symptom Checklist-90-Revised (SCL-90-R). Pearson, 1975. 